\title{
Existence and Nash implementation of efficient sharing rules for a commonly owned technology
}

\author{
Luis C. Corchón ${ }^{1}$, M. Socorro Puy ${ }^{2}$ \\ 1 Departamento de Economía, Universidad Carlos III, 28903 Getafe (Madrid), Spain \\ (e mail: 1corchon@eco.uc3m.es) \\ ${ }^{2}$ Departamento de Teoría e Historia Económica, Universidad de Málaga, \\ Campus el Ejido, 29013 Málaga, Spain (e mail: mps@uma.es)
}

Received: 29 June 1998/Accepted: 15 November 2000

\begin{abstract}
Suppose that a group of individuals owns collectively a technology which produces a consumption good by means of a (possibly heterogeneous) input. A sharing rule associates input contributions with a vector of consumptions that are technologically feasible. We show that the set of allocations obtained by any continuous sharing rule contains Pareto efficient allocations. We also present a mechanism that implements in Nash equilibrium the Pareto efficient allocations contained in an arbitrary sharing rule.
\end{abstract}

\section{Introduction}

Consider a group of people owning a technology which transforms a possibly heterogeneous input (labor) into a homogeneous output (consumption). Inputs are also provided by the owners. Different proposals on how to distribute the output can be found in the literature.

In the class of environments in which the input is homogenous, Roemer and Silvestre (1988) proposed the Proportional Solution and the Equal Benefit Solution and Mas-Colell (1980) proposed the Constant Returns Equivalent Solution. Several characterizations of these solutions are provided in Moulin (1990), Moulin and Roemer (1989), and Maniquet (1996). When heterogenous inputs are considered, other solutions have been proposed: Equal Sharing,

We are grateful to Jose Alcalde, Carmen Beviá, Matt Jackson, Jörg Naeve, Francçois Maniquet, Ignacio Ortuño Ortín, Martin Peitz, Murat Sertel, Joaquin Silvestre, two anonymous referees and the seminar audience of the University of Alicante for very helpful comments on a preliminary version of this paper. We are also grateful to John Roemer for the initial stimulus that led us to write this note. Of course we are fully responsible for any remaining error. This research has been partially financed by CICYT PB 1998 0024. We thank finally financial support from the IVIE. 
Marginal Cost Rule, Aumann-Shapley Prices, Reference Welfare Equivalent Budget, etc. (see Aumann and Shapley 1974; Billera and Heath 1982; Moulin 1987; Tauman 1988; Pfingsten 1991 and Fleurbaey and Maniquet 1996).

In this paper we focus our attention on contracts that are offered to the owners of the inputs. We assume that the quantity of inputs is contractible but preferences are not. These contracts, that we will call Sharing Rules, are a function that specifies the list of consumptions depending on input contributions. The sharing rule together with the quantity of inputs determine income distribution inside the firm (the idea of expressing the share of output in terms of the inputs first appeared in Moulin 1990, p. 445). It is worth to notice that all solutions mentioned above qualify as Sharing Rules except the constant return equivalent solution. Our analytical task consists in checking which Sharing Rules satisfy two basic requirements: Efficiency and Implementability.

Firstly, we focus attention on those Sharing Rules which are compatible with Pareto efficiency, in the sense that there are allocations which are Pareto efficient and the input-output combination belongs to the sharing rule. We call those sharing rules Efficient Sharing Rules. It is well known that the proportional and the equal benefit solutions are Efficient Sharing Rules. We generalize these results by showing that any continuous sharing rule is an efficient sharing rule in the set of classical economies (continuous and convex preferences). Our proof is inspired by the proof of Negishi (1960) of the existence of a Walrasian Equilibrium.

Secondly, we consider the incentive properties of efficient sharing rules. A sharing rule that gives incentives to distort preferences or productivities can not be regarded as satisfactory. Roemer (1988), Gevers (1986) and Maniquet and Fleurbaey (1996) showed respectively that the proportional, the equal benefit and the reference welfare equivalent budget efficient sharing rules are Nash implementable, ${ }^{1}$ i.e. there is a mechanism whose Nash equilibrium strategies generate the desired allocations. Suh (1995) introduced a mechanism that implements the proportional solution in Nash, undominated Nash and Strong equilibria. Shin and Suh (1997) provide a simple mechanism which doubly implements a class of solutions in Nash and strong equilibrium. In this paper we provide a simple mechanism that implements in Nash equilibrium every efficient sharing rule in the set of classical economies when there are at least three individuals. ${ }^{2}$ We assume that the planner knows the sharing rule but not the preferences of the agents that determine the set of Pareto efficient allocations for each economy. Our procedure has the advantage over Shin and Suh's that our conditions on the solutions to be implemented are easy to check and they include economies with heterogeneous inputs, but the disadvantage that we only implement in Nash equilibrium.

In our mechanism, people are arranged in a circle and each agent proposes

\footnotetext{
1 Implementation in dominant strategies is usually impossible. However see Schmei dler and Tauman (1989) for a case in which it is possible.

${ }^{2}$ We consider adverse selection problems only. See Holmstrom (1982) for problems of moral hazard.
} 
the amount of input supplied by him and the agent next to him. Three cases are then identified:

First, when the amount of input proposed by each agent coincides with the amount suggested by his monitor. In this case the mechanism distributes the output according to the sharing rule.

Second, when there are, at most, two consecutive agents whose proposals differ from what was proposed for them. Then, the agent with the lowest index (the dissident) has the right to choose an allocation in a certain budget set that is only profitable if he has deviated from a non efficient allocation. Since a deviation can only happen if the monitor of the dissident has tried to fool the mechanism, the monitor is severely fined: he gets zero consumption and has to contribute with the maximum amount of labor. All other agents obtain some arbitrary bundle.

Third, for any other message it is not possible to identify the dissident. In this case, the mechanism divides the agents into two groups: the ones that consume but do not work, and the ones that work but do not consume. Notice that, contrarily to what happens in the canonical mechanism, agents do not play integer games.

Our construction avoids some of the criticism made by Jackson (1992) to canonical mechanisms, since the strategy sets are compact.

\section{The model and the main results}

There is one consumption good produced from a vector of possibly heterogenous inputs using a publicly owned technology.

There are $n$ individuals indexed by $i$. Let $N=\{1, \ldots, n\}$. They are endowed with $\bar{\ell} \in \mathbb{R}_{+}$, units of input. ${ }^{3}$ Each individual consumption set is defined by:

$$
\mathbb{X}=\left\{\left(x_{i}, \ell_{i}\right): x_{i} \in \mathbb{R}_{+}, \ell_{i} \in[0, \bar{\ell}]\right\}
$$

where $x_{i}$ is agent $i$ 's consumption and $\ell_{i}$ is input contribution. Each agent has preferences defined on $\mathbb{X}$, that can be represented by a utility function

$$
u_{i}: \mathbb{X} \rightarrow \mathbb{R}
$$

The utility function is assumed to be differentiable, concave, strictly increasing in $x_{i}$ and strictly decreasing in $\ell_{i}$. Thus

$$
\underset{\left(x_{i}, \ell_{i}\right) \in \mathbb{X}}{\arg \min } u_{i}\left(x_{i}, \ell_{i}\right)=(0, \bar{\ell}) \quad \forall i \in N .
$$

The technology is represented by a production function

$$
f: \mathbb{R}_{+}^{n} \rightarrow \mathbb{R}_{+}
$$

The function $f$ is continuous, increasing in each component, concave, continuously differentiable in each component and with $f(0, \ldots, 0)=0$. 3 When inputs are heterogenous, we assume that inputs are normalized so that the
quantity held by each agent is the same. 
We define the feasible set, denoted by $\mathscr{X}$ as follows

$$
\mathscr{X}=\left\{\left(x_{1}, \ell_{1}, \ldots, x_{n}, \ell_{n}\right) \in \mathbb{X}^{n}: \sum_{i \in N} x_{i} \leq f\left(\ell_{1}, \ldots, \ell_{n}\right)\right\}
$$

A feasible allocation is denoted by $(x, \ell) \in \mathscr{X}$ where $x=\left(x_{1}, \ldots, x_{n}\right)$ and $\ell=$ $\left(\ell_{1}, \ldots, \ell_{n}\right)$.

We assume that $\mathscr{X}$ is fixed and utility functions vary. Thus, an economy, denoted by $u=\left(u_{1}, \ldots, u_{n}\right)$, is a list of utility functions satisfying the assumptions listed above. The set of admissible economies is denoted by $\mathscr{E}$.

The Pareto efficient solution $\varphi^{E}: \mathscr{E} \rightarrow \mathscr{X}$ associates to each economy in the domain the set of Pareto efficient allocations for this economy. Formally,

$$
\varphi^{E}(u)=\left\{\begin{array}{c}
(x, \ell) \in \mathscr{X}: \nexists\left(x^{\prime}, \ell^{\prime}\right) \in \mathscr{X} / u_{h}\left(x_{h}^{\prime}, \ell_{h}^{\prime}\right) \geq u_{h}\left(x_{h}, \ell_{h}\right) \forall h \in N \\
\text { and } u_{j}\left(x_{j}^{\prime}, \ell_{j}^{\prime}\right)>u_{j}\left(x_{j}, \ell_{j}\right) \text { for at least one } j \in N
\end{array}\right\} .
$$

A Sharing Rule is a contract that specifies the consumptions as a function of input contributions. Formally, a sharing rule $P=\left(P_{1}, \ldots, P_{n}\right)$ is a collection of functions such that $P_{i}:[0, \bar{\ell}]^{n} \rightarrow \mathbb{R}_{+} \forall i \in N$ with $\sum_{i \in N} P_{i}(\ell)=f(\ell) \forall \ell \in$
$[0, \bar{\ell}]^{n}$.

Each $P_{i}$ yields the consumption of $i$ as a function of $\ell$. Moreover, $P$ distributes the total output. Some examples of solutions that can be expressed as sharing rules are the following:

The Proportional Solution in which the sharing rule is:

$$
P_{i}(\ell)=\frac{f(\ell)}{\sum_{i \in N} \ell_{i}} \ell_{i} \quad \forall i \in N,
$$

where the amount of output consumed by an agent is proportional to the amount of input that he contributes.

The Equal Benefit Solution, in which the sharing rule is:

$$
P_{i}(\ell)=\frac{\partial f(\ell)}{\partial \ell_{i}} \ell_{i}+\frac{1}{n}\left[f(\ell)-\sum_{i \in N}\left(\frac{\partial f(\ell)}{\partial \ell_{i}} \ell_{i}\right)\right] \quad \forall i \in N,
$$

where each agent consumes according to the budget constraint in the Walrasian equilibrium with equal profits. Clearly, other rules of profit distribution also qualify as sharing rules.

The Equal Sharing Solution, in which the sharing rule is:

$$
P_{i}(\ell)=\frac{f(\ell)}{n} \quad \forall i \in N,
$$

where each agent consumes an equal part of the total output.

The Aumann-Shapley prices, in which for a vector of heterogenous input contributions, the sharing rule is:

$$
P_{i}(\ell)=\int_{0}^{1} \frac{\partial f(t \ell)}{\partial \ell_{i}} d t \ell_{i} \quad \forall i \in N,
$$


where each agent consumes proportionally to the contribution of his input to the total production. When the input is homogenous it coincides with the proportional sharing rule.

Furthermore, the family of methods proposed by Moulin (1987) also qualify as sharing rules. These sharing rules are:

For each $\mu \in \mathbb{R}_{+}$,

$$
\begin{aligned}
P_{i}(\ell)= & \frac{\ell_{i}}{\sum_{i \in N} \ell_{i}} f(\ell) \quad \text { if } \frac{f(\ell)}{\sum_{i \in N} \ell_{i}} \leq \mu \\
& \ell_{i} \mu+\frac{1}{n}\left(f(\ell)-\mu \sum_{i \in N} \ell_{i}\right) \quad \text { if } \frac{f(\ell)}{\sum_{i \in N} \ell_{i}} \geq \mu \quad \forall i \in N .
\end{aligned}
$$

For each $\lambda \in[0,1]$,

$$
P_{i}(\ell)=\frac{f(\ell)}{n}+\left(\ell_{i}-\frac{1}{n} \sum_{i \in N} \ell_{i}\right)\left[\left(1+\frac{f(\ell)}{\sum_{i \in N} \ell_{i}}\right)^{\lambda}-1\right] \quad \forall i \in N .
$$

For each $\lambda \in[0,1]$,

$$
P_{i}(\ell)=\frac{\ell_{i}^{\lambda}}{\sum_{i \in N} \ell_{i}^{\lambda}} f(\ell) \quad \forall i \in N .
$$

For each $\lambda \in[0,1)$,

$$
\left.P_{i}(\ell)=\left(\ell_{i}^{1} \lambda+\alpha\right)^{1 /(1} \lambda\right)-\ell_{i} \quad \forall i \in N,
$$

where $\alpha$ is the unique solution to: $\left.\sum_{i \in N}\left(\ell_{i}^{1} \lambda+\alpha\right)^{1 /(1} \lambda\right)=\sum_{i \in N} \ell_{i}+f(\ell)$.

Finally, note that every convex combination of the mentioned solutions, is also a sharing rule.

We assume that every sharing rule verifies that if $\ell_{i}=\bar{\ell}, P_{i}\left(\ell_{1}, . . \bar{\ell}, \ell_{i+1}, . . \ell_{n}\right)$ $>0$. Note that all the mentioned sharing rules satisfy this assumption.

\subsection{Pareto efficiency and Sharing Rules}

In the sequel we will be interested in the intersection between the Pareto efficient allocations $\varphi^{E}$ and those satisfying a sharing rule $P$. Those allocations will be called Efficient Sharing Rules and are denoted by $\varphi^{P E}$ where:

$$
\varphi^{P E}(u, P)=\left\{(x, \ell) \in \varphi^{E}(u): x_{i}=P_{i}(\ell), \forall i \in N\right\} .
$$

We now prove that $\varphi^{P E}(u, P) \neq \varnothing$, provided that $P$ is continuous.

Theorem 1. Given $u \in \mathscr{E}$ and a continuous sharing rule $P$, then $\varphi^{P E}(u, P) \neq \varnothing$.

Proof. Let $\alpha \in \Delta^{n 1}$ where $\Delta^{n} 1=\left\{\alpha \in \mathbb{R}_{+}^{n}: \sum_{i \in N} \alpha_{i}=1\right\}$.
$\quad$ Consider the problem:

$$
\max _{(x, \ell) \in \mathscr{X}} \sum_{i \in N} \alpha_{i} u_{i}\left(x_{i}, \ell_{i}\right)
$$


by continuity of $u_{i}$ and compactness of $\mathscr{X}$, there always exists a solution to this problem, which is by definition Pareto efficient.

This maximization defines a correspondence denoted by $\phi$, such that

$\phi: \Delta^{n}{ }^{1} \rightarrow \mathscr{X}$

By concavity of $u$ and convexity of $\mathscr{X}, \phi$ is convex valued. By Berge's maximum theorem, $\phi$ is upper hemicontinuous.

We define $D_{i}: \mathbb{R}_{+} \times[0, \bar{\ell}]^{n} \rightarrow \mathbb{R}$ for each $i \in N$ as follows:

$D_{i}\left(x_{i}, \ell\right)=P_{i}(\ell)-x_{i}$.

Fix a feasible allocation $(\hat{x}, \hat{\ell})$ and consider the following maximization program:

$$
\max _{\alpha \in \Delta^{n}} \sum_{i \in N} \alpha_{i} D_{i}\left(\hat{x}_{i}, \hat{\ell}\right)
$$

by compactness of $\Delta^{n}{ }^{1}$ and continuity of the objective function on $\alpha_{i}$, there always exists a solution to this problem. This maximization defines a correspondence denoted by $\Phi$, such that

$$
\Phi: X \rightarrow \Delta^{n} 1
$$

where $\Phi$ is convex valued and upper hemicontinuous by Berge's maximum Theorem (remember that each $D_{i}$ is continuous on $\left(x_{i}, \ell_{i}\right)$ ).

Now consider the following mapping

$$
\Phi \times \phi: \Delta^{n}{ }^{1} \times \mathscr{X} \rightarrow \Delta^{n}{ }^{1} \times \mathscr{X} \text {. }
$$

This is an upper hemicontinuous mapping from a compact convex set into itself, with non empty and convex values. By Kakutani's fixed point Theorem, there exists a fixed point $\left(\alpha^{*}, x^{*}, \ell^{*}\right)$.

Notice that it is impossible to have $\left(x_{i}^{*}, \ell^{*}\right)$ such that $D_{i}\left(x_{i}^{*}, \ell^{*}\right)<0 \forall i \in N$ or $D_{i}\left(x_{i}^{*}, \ell^{*}\right)>0 \forall i \in N$, because the sharing rules verify that $\sum_{i \in N} x_{i}=f(\ell)$. Thus, if $D_{i}\left(x_{i}^{*}, \ell^{*}\right)>0$, then $\exists j \in N: D_{j}\left(x_{j}^{*}, \ell^{*}\right)<0$ and so $\alpha_{j}^{*}=0$. But this implies that $\phi$ will assign $\left(x_{j}^{*}, \ell_{j}^{*}\right)=(0, \bar{\ell})$ and so $D_{j}\left(x_{j}^{*}, \ell^{*}\right) \geq 0$, a contradiction. Therefore, the fixed point verifies $D_{i}\left(x_{i}^{*}, \ell^{*}\right)=0 \forall i \in N$ and this implies that $x_{i}^{*}=P_{i}(\ell) \forall i \in N$.

It can be easily checked that under our assumptions all the rules mentioned before are continuous (for Aumann-Shapley prices, see Mirman and Tauman 1980). Thus, those sharing rules are efficient sharing rules.

\subsection{Implementation of Efficient Sharing Rules}

A mechanism $\Gamma$ is a list $\left\{\left(S_{i}\right)_{i \in N}, g\right\}$ where $S_{i}$ is the strategy space for agent $i$ and $g$ is the outcome function, mapping each strategy profile into an element of the feasible set:

$$
g: \prod_{i \in N} S_{i} \rightarrow \mathscr{X}
$$

The outcome received by each agent is $g_{i}(s)=\left(x_{i}, \ell_{i}\right)$. 
Let $s_{i}$ be the list of strategies for all the agents except for $i$, then, the set of Nash equilibria of the game $(\Gamma, u)$ is denoted by $\operatorname{NE}(\Gamma, u)$.

$$
N E(\Gamma, u)=\left\{s \in \prod_{i \in N} S_{i}: u_{i}\left(g_{i}(s)\right) \geq u_{i}\left(g_{i}\left(s_{i}^{\prime}, s_{i}\right) \forall i \in N, \forall s_{i}^{\prime} \in S_{i}\right)\right\} .
$$

We say that a mechanism implements $\varphi^{P E}$ in Nash equilibrium when it verifies that:

$$
\begin{aligned}
& N E(\Gamma, u) \neq \varnothing \\
& \varphi^{P E}(u, P)=g(N E(\Gamma, u)), \quad \forall u \in \mathscr{E} .
\end{aligned}
$$

For each continuous sharing rule $P$, let the mechanism $\Gamma(P)$ be as follows:

The strategy space of agent $i$ is $S_{i}=[0, \bar{\ell}]^{2} \subset \mathbb{R}_{+}^{2}$.

A strategy for $i$ is a pair, $s_{i}=\left(\ell_{i}^{i}, \ell_{i}^{i+1}\right)$, when $i=n$ we define $i+1=1$ and when $i=1, i-1=n$. Each individual strategy may be interpreted as a proposed labor allocation for himself $\ell_{i}^{i}$ and the individual next to $\operatorname{him} \ell_{i}^{i+1}$. This is a particular instance of a "Tweed Ring" mechanism. ${ }^{4}$

The outcome function is divided into three cases which we denote as rules:

Rule 1 (Unanimity). If $\forall j \in N: \ell_{j}^{j}=\ell_{j 1}^{j}$ then $g_{j}(s)=\left(P_{j}(\ell), \ell_{j}^{j}\right)$ where $\ell=\left(\ell_{1}^{1}, \ldots, \ell_{n}^{n}\right)$.

Rule 2 (Dissident right). If $\forall j \in N-\{i\}: \ell_{j}^{j}=\ell_{j}^{j}$ and for $i: \ell_{i}^{i} \neq \ell_{i 1}^{i}$, or if $\forall j \in N-\{i, i+1\}: \ell_{j}^{j}=\ell_{j}^{j}$ and for $i$ and $i+1: \ell_{i}^{i} \neq \ell_{i}^{i}$ and $\ell_{i}^{i+1} \neq \ell_{i+1}^{i+1}$.

Agent $i$ is called the dissident, agent $i-1$ is the punished agent and the rest are denoted by $k$. Let $x_{i}=P_{i}\left(\ell^{\prime}\right)+\frac{\partial f\left(\ell^{\prime}\right)}{\partial \ell_{i}}\left(\ell_{i}^{i}-\ell_{i}^{i}{ }_{1}\right)$ with $\ell^{\prime}=\left(\ell_{1}^{1}, ., \ell_{i}^{i} 11\right.$
$\left.\ell_{i 1}^{i}, \ell_{i+1}^{i+1}, ., \ell_{n}^{n}\right)$ then,

$$
\begin{aligned}
& g_{i}(s)=\left(x_{i}, \ell_{i}^{i}\right), \quad g_{i} 1(s)=(0, \bar{\ell}), \\
& g_{k}(s)=\left(\frac{f\left(\ell_{1}^{1}, ., \ell_{i}^{i} 2 \frac{2}{2}, \bar{\ell}, \ell_{i}^{i}, ., \ell_{n}^{n}\right)-x_{i}}{n-2}, \ell_{k}^{k}\right) .
\end{aligned}
$$

And only in the case that from this rule $x_{i}>f\left(\ell_{1}^{1}, ., \ell_{i}^{i} 2 \frac{2}{2}, \bar{\ell}, \ell_{i}^{i}, ., \ell_{n}^{n}\right)$ then,

$$
g_{i}(s)=\left(f\left(\ell_{1}^{1}, ., \ell_{i}^{i} \frac{2}{2}, \bar{\ell}, \ell_{i}^{i}, ., \ell_{n}^{n}\right), \ell_{i}^{i}\right), \quad g_{i} 1(s)=(0, \bar{\ell}), \quad g_{k}(s)=\left(0, \ell_{k}^{k}\right) .
$$

Rule 3. It applies when we are not in Rule 1, nor in Rule 2.

Let $M=\left\{i \in N: s_{i}=(0,0)\right\}$ and let $\hat{\ell}=\left(\hat{\ell}_{1}, \ldots, \hat{\ell}_{n}\right)$ where $\forall i \in M, \hat{\ell}_{i}=0$ and $\forall j \in N-M, \hat{\ell}_{j}=\max \left\{\frac{\bar{\ell}}{\beta}, \min \left\{\beta \ell_{j}^{j+1}, \bar{\ell}\right\}\right\}$ with $\beta>1$, then $\forall i \in M$ : $g_{i}(s)=\left(\frac{f(\hat{\ell})}{\# M}, 0\right)$ and for $\forall j \in N-M: g_{j}(s)=\left(0, \hat{\ell}_{j}\right)$.

The interpretation of this mechanism is the following:

In Rule 1 each individual is given the share of the total output according to the sharing rule $P$.

Rule 2 applies when one individual deviates. Notice that the maximization problem for the dissident agent is

4 Another example of a "Tweed Ring" mechanism is due to M. Walker (1981). 


$$
\max _{\ell_{i}^{i} \in[0, \bar{\ell}]} u_{i}\left(P_{i}\left(\ell^{\prime}\right)+\frac{\partial f\left(\ell^{\prime}\right)}{\partial \ell_{i}}\left(\ell_{i}^{i}-\ell_{i}^{i} 1\right), \ell_{i}^{i}\right)
$$

considering interior solutions, the first order condition to this problem is a necessary condition for an efficient allocation:

$$
\frac{\partial u_{i} / \partial x_{i}}{\partial u_{i} / \partial \ell_{i}}=\frac{-1}{\partial f\left(\ell^{\prime}\right) / \partial \ell_{i}}
$$

and it is sufficient if this equality is verified for every agent. Thus, if the announcement of the rest of agents does not lead to a Pareto efficient allocation, one individual can increase his payoff deviating. ${ }^{5}$ Also, Rule 2 punishes the individual who did not monitor adequately. The punished individual is given his worst allocation. If the consumption proposed by the dissident is not feasible, we give him all the output.

In Rule 3, the mechanism divides the agents into two groups: Those announcing $s_{i}=(0,0)$ who consume and do not work, and those announcing $s_{i} \neq(0,0)$ who work but do not consume. As we next show, there is always an agent of this second group that strictly improves by means of a deviation.

Theorem 2. If $n \geq 3$, the mechanism $\Gamma(P)$ implements $\varphi^{P E}(u, P)$ in Nash equilibrium.

Proof. First, let us show that $\varphi^{P E}(u, P) \subseteq g(N E(\Gamma(P), u)) \forall u \in \mathscr{E}$.

Let $(x, \ell) \in \varphi^{P E}(u, P)$ for some $u \in \mathscr{E}$ and some $P$, let $s \in \prod_{i \in N} S_{i}$ be a strategy profile defined by $s=\left(s_{1}, \ldots, s_{n}\right)$ and $s_{i}=\left(\ell_{i}^{i}, \ell_{i}^{i+1}\right)$ such that $\ell_{i}^{i}=\ell_{i 1}^{i} \forall i \in N$. Then, the outcome induced by $s$ is $g(s)=(x, \ell)$. We verify that $s \in N E(\Gamma(P), u)$.

If $(x, \ell) \in \varphi^{E}(u)$ is an interior solution, then $\forall i, \ell_{i}^{i}$ verifies first order optimality conditions:

$$
\frac{\partial u_{i} / \partial x_{i}}{\partial u_{i} / \partial \ell_{i}}=\frac{-1}{\partial f\left(\ell^{\prime}\right) / \partial \ell_{i}} \quad \forall i \in N
$$

and so, if one agent deviates, Rule 2 applies and the dissident agent can not get anything preferred to $\left(x_{i}, \ell_{i}^{i}\right)$. Even if $(x, \ell) \in \varphi^{E}(u)$ is a non interior solution, there is no dissident agent which can get anything preferred to $\left(x_{i}, \ell_{i}^{i}\right)$. Therefore, $s \in N E(\Gamma(P), u)$.

Second, let us show that $g(N E(\Gamma(P), u)) \subseteq \varphi^{P E}(u, P) \forall u \in \mathscr{E}$.

Let $s \in N E(\Gamma(P), u)$ and $g(s)=(x, \ell)$.

Case 1. When $(x, \ell)$ is in Rule 1 but $(x, \ell) \notin \varphi^{E}(u)$. Then, $\exists\left(x^{\prime}, \ell^{\prime}\right) \in \mathscr{X}$ such that at least one agent is better off. If this agent deviates, Rule 2 applies. Let $\ell^{\prime}=\left(\ell_{1}^{1}, \ldots, \ell_{i}^{i} 1, \ell_{i}^{i} 1, \ell_{i+1}^{i+1}, \ldots, \ell_{n}^{n}\right)$. The attainable set for this agent is denoted by $\mathbb{A}_{i}$, where

${ }^{5}$ It can be shown that for non interior solutions, Rule 2 also gives incentives to deviate from a non efficient allocation, since individuals can trade leisure for consumption at a rate equal to the marginal rate of transformation. 


$$
\mathbb{A}_{i}=\left\{\left(x_{i}, \ell_{i}\right) \in \mathbb{X}: x_{i}=P_{i}\left(\ell^{\prime}\right)+\frac{\partial f\left(\ell^{\prime}\right)}{\partial \ell_{i}}\left(\ell_{i}-\ell_{i}^{i}\right)\right\} .
$$

Since $(x, \ell)$ is not efficient, there is at least one agent for whom the marginal rate of substitution is greater (or lower) than the marginal rate of transformation. Then, announcing a lower (respectively higher) input contribution, he improves his payoff. This contradicts that $s \in N E(\Gamma(P), u)$.

Case 2. $(x, \ell)$ Comes from Rule 2. The punished individual $i-1$ receives $g_{i} 1(s)=(0, \bar{\ell})$ and let us then show that a deviation in the announcement of agent $i-1$ can increase his payoff:

When $\ell_{j}^{j}=\ell_{j 1}^{j} \forall j \in N-\{i\}$, then a deviation of agent $i-1$ announcing $\ell_{i}^{\prime i}=\ell_{i}^{i}$ leads to the unanimity rule. In the case that $\ell_{i 1}^{\prime}=\bar{\ell}$, we have assumed that $P_{i}{ }_{1}\left(\ell_{1}, ., \bar{\ell}, \ell_{i}, \ldots, \ell_{n}\right)>0$, so that agent $i-1$ will increase his payoff.

When $\ell_{j}^{j}=\ell_{j 1}^{j} \forall j \in N-\{i, i+1\}$, a deviation of agent $i-1$ such that $s_{i 1}^{\prime}=\left(\ell_{i 1_{1}{ }^{\prime}}^{\prime}, \ell_{i 1}^{\prime i}\right)$ where $\ell_{i 1}^{\prime i} 1 \neq \ell_{i 2}^{i}{ }_{2}^{1}$ and $\ell_{i 1}^{\prime i} \neq \ell_{i}^{i}$, will lead to Rule 3 . In Rule 3 we have that for all $\ell_{i}^{\prime i} \neq \bar{\ell}$, agent $i-1$ can increase his payoff.

We conclude therefore, that there is no Nash equilibrium in Rule 2.

Case 3. $(x, \ell)$ Comes from Rule 3. Then, a deviation in one of the agents strategy, can move the outcome either to Rule 2 (Case 3.1) or to Rule 3 (Case 3.2).

Case 3.1. We then have that $\# N-M \geq 2$. Consider a deviation of agent $j \in$ $N-M, s_{j}^{\prime}=\left(\ell_{j 1}^{j}, \ell_{j+1}^{j+1}\right)$ where $\ell_{j 1}^{j}$ and $\ell_{j+1}^{j+1}$ are respectively part of the equilibrium strategies of the agents $j-1$ and $j+1$. This deviation moves the outcome to Rule 2, where notice that agent $j$ can not be the punished agent nor the dissident. Therefore, he obtains the payoff $\left(x_{j}^{\prime}, \ell_{j}^{j} 1_{j}\right)$ where $x_{j}^{\prime}$ is calculated according to Rule 2 . Let us next show that $u_{j}\left(x_{j}^{\prime}, \ell_{j 1}^{j}\right)>u_{j}\left(0, \hat{\ell}_{j}\right)$, i.e., that the proposed deviation strictly improves agent $j$. It is sufficient to show that $\ell_{j 1}^{j}<\hat{\ell}_{j}$ : If $j-1 \in N, \ell_{j 1}^{j}=0$ and since $\hat{\ell}_{j}=\max \left\{\frac{\bar{\ell}}{\beta}, \min \left\{\beta \ell_{j}^{j+1}, \bar{\ell}\right\}\right\}>0$, it is sat-
isfied.

If $j-1 \in M-N, \ell_{j 1}^{j}$ such that $\beta \ell_{j 1}^{j} \geq \bar{\ell}$, can not be part of an equilibrium strategy because then $\min \left\{\beta \ell_{j 1}^{j}, \bar{l}\right\}=\bar{\ell}$, and so $\hat{\ell}_{j}{ }_{1}=\bar{\ell}$. Therefore, in equilibrium $\ell_{j 1}^{j}<\frac{\bar{\ell}}{\beta}$. Furthermore, $\ell_{j 1}^{j}$ such that $\beta \ell_{j 1}^{j}>\frac{\bar{\ell}}{\beta}$ can neither be part of an equilibrium strategy because then $\hat{\ell}_{j} 1=\max \left\{\frac{\bar{\ell}}{\bar{\beta}}, \beta \ell_{j}^{j} 1\right\}=\beta \ell_{j}^{j}$ and agent $j-1$ will increase his payoff announcing $\ell_{j}^{j} \leq \frac{\bar{\ell}}{\beta^{2}}$. Since for all the agents $j \in M-N$ the above argument applies, we then have that $\hat{\ell}_{j}=\frac{\bar{\ell}}{\beta}$, and so $\ell_{j 1}^{j}<\hat{\ell}_{j}$.

Case 3.2. Consider a deviation of agent $j \in M-N$ to $s_{j}^{\prime}=(0,0)$. This deviation, clearly, improves agent $j$ outcome. 
We conclude therefore, that there is no Nash equilibrium in Rule 3.

The proposed mechanism satisfies some nice properties: The message space consists of announcements of input contributions. Furthermore, participants contribute, in equilibrium, with the amount they announce (a similar property is called Forthrightness by Saijo et al. 1996).

\section{Final comments}

In this paper we have shown that any continuous sharing rule is compatible with efficiency and incentives. In this sense, our results suggest the existence of a large degree of freedom concerning income distribution within the firm, unless other consideration are introduced. We would like to remark that shirking by workers is never reported as a concern in studies of cooperatives in the real world: observers report that workers monitor each other successfully (see Bonin et al. 1993). This is precisely what happens in our mechanism.

Finally, see Corchón and Puy (1998) for a study of sharing rules yielding individually rational allocations and sharing rules that arise from voting inside the firm.

\section{References}

Aumann RJ, Shapley LS (1974) Values of non atomic games. Princeton University Press, Princeton

Billera LJ, Heath DC (1982) Allocation of Shared Costs: A Set of Axioms Yielding a Unique Procedure. Math Methods Oper Res 7: 32 39

Bonin J, Jones D, Putterman L (1993) Theoretical and empirical studies of producer cooperatives: Will ever the twain meet? J Econ Lit 31: 12901320

Corchón LC, Puy MS (1998) Individual rationality and voting in cooperative produc tion. Econ Lett 59: 8390

Fleurbaey M, Maniquet F (1996) Fair allocation with unequal production skills: The no envy approach to compensation. Math Soc Sci 32: 7193

Gevers L (1986) Walrasian social choice: Some simple axiomatic approaches. In: Heller W et al. (eds) Social choice and public decision making: Essays in Honor of K. J. Arrow. Cambridge University Press, New York, 1: 97114

Holmstrom B (1982) Moral Hazard. Bell J Econ 13 (2): 324340

Jackson MO (1992) Implementation in undominated strategies: A look at bounded mechanisms. Rev Econ Stud 59: 757775

Maniquet F (1996) Allocation rules for a commonly owned technology: The average cost lower bound. J Econ Theory 69: 490507

Mas Colell A (1980) Remarks on the game theoretic analysis of a simple distribution of surplus problem. Int J Game Theory 9: 125140

Mirman LJ, Tauman LJ (1980) The continuity of the Aumann Shapley Price Mecha nism. J Math Econ 9: 235249

Moulin H (1987) Equal or proportional division of a surplus, and other methods. Int $\mathbf{J}$ Game Theory 16: 161186

Moulin H (1990) Joint ownership of a convex technology: Comparison of three solu tions. Rev Econ Stud 57: 439452 
Moulin H (1995) Cooperative Microeconomic. A game theoretic introduction. Prince ton University Press, Princeton

Moulin H, Roemer JE (1989) Public ownership of the external world and private ownership of shelf. J Polit Econ 97: 347367

Negishi T (1960) Welfare economics and the existence of an equilibrium for a com petitive economy. Metroecon 12: 9297

Pfingsten A (1991) Surplus sharing methods. Math Soc Sci 21: 287301

Roemer JE (1988) A public ownership resolution of the tragedy of the commons. Mimeo, California

Roemer JE (1996) Theories of distributive justice, Chapt. 6. Harvard University Press, Cambridge, Massachusetts, London, England

Roemer JE, Silvestre J (1988) Public ownership: Three proposals for resource alloca tion. Mimeo, University of California

Roemer JE, Silvestre J (1993) The proportional solution for economies with both pri vate and public ownership. J Econ Theory 59(2): 426444

Saijo T, Tatamitani Y, Yamato T (1996) Toward natural implementation. Int Econ Rev 37: 949980

Saijo T (1988) Strategy space reduction in Maskin's theorem: Sufficient conditions for Nash implementation. Econometrica 56: 693700

Shin S, Suh SC (1997) Double implementation by a simple game form in the commons problem. J Econ Theory 77: 205213

Schmeidler D, Tauman Y (1989) Incentive compatible cost allocation schemes. IMW, Working paper n. 173. University of Bielefeld, Germany

Suh SC (1995) A mechanism implementing the proportional solution. Econ Design 1: 301317

Tauman Y (1988) The Aumann Shapley prices: A survey. The Shapley value: Essays in honor of LLoyd S. Shapley. Cambridge University Press, Cambridge New York and Melbourne pp 279304

Walker M (1981) A simple incentive compatible scheme for attaining Lindahl alloca tions. Econometrica 49: 6573 